\title{
Roving Survey for the Incidence of Tip Over Disease of Banana in Major Banana Growing Districts of Karnataka, India
}

\author{
N. Pradnyarani*, M.S. Kulkarni, K.C. Kirankumar, R.K. Mesta, \\ B. Jagadeesh and H. Rohan
}

Department of Plant Pathology, College of Horticulture, Bagalkot, University of Horticultural Sciences, Bagalkot, Karnataka, India

*Corresponding author

\section{A B S T R A C T}

\section{Keywords}

Banana, Tip over disease, Bacteria, Disease incidence, loss

Article Info

Accepted:

10 June 2018

Available Online:

10 July 2018
The survey was undertaken during 2015-16 in the tip over disease infected areas of banana. The disease incidence was found to be the highest in Bagalkot district $(49.67 \%)$ followed by Belagavi (34.16\%), Dharwad (25.75\%), Vijayapura (24.81\%), Bidar (15.75\%), Haveri (13.24\%), Koppal (11.07\%), Mysore (10.72\%) and the least was noticed in Gadag district $(8.71 \%)$. The severity of disease was found to be more in tissue culture raised plantlets. The per cent of incidence of tip over disease ranged from 0.40 (Hoovinahal village of Koppal taluk) to 99.27 per cent (Kamalnagar village of Bidar district) in the field. The infection occurred on new banana plantations of one to six month age. Banana plantations of 8 to 10 weeks age were found to be severely infected.

\section{Introduction}

Banana is known for its antiquity interwoven with Indian heritage, culture and is considered as a symbol of 'Prosperity and fertility'. Owing to its greater socio-economic significance and multifaceted uses, it is referred as Kalpataru (Plant of virtue). Botanically, banana fruit is a wonder berry which forms the staple food of millions of peoples across the globe providing more balanced food than any other fruit or vegetable. Banana is one of the biggest single trade items in international fruit trade (Snehalatharani and Khan, 2009). Banana is a nutritious, palatable and easily digestible fruit and remains available throughout the year.

The banana crop is affected by many diseases around the world. Tip-over disease of banana, which was considered to be minor earlier, has assumed serious proportions in recent years in India in general and Karnataka in particular. With the advent of tissue culture technique for the mass production of banana plants in view of fulfilling increased demand due to the rapid expansion of banana cultivation, the disease is spreading fast causing high plant mortality and consequent losses to the planters. With regular field visits and farmers feedback noticed that 
the disease is more in almost all growing areas especially in tissue cultured banana plants. Tip- over is a serious problem especially in tissue cultured banana plants and the disease incidence ranges from 5.05 to 65.28 per cent (Vijayalaxmi et al., 2014) in several parts of the banana growing areas next to moko disease. Hence, the present investigation was undertaken to know the incidence of the disease in major banana growing districts of Karnataka.

\section{Materials and Methods}

Intensive roving survey was conducted during 2015-2016 to know the disease incidence in major banana growing districts of Karnataka viz., Bagalkot, Belagavi, Bidar, Dharwad, Gadag, Haveri, Koppal, Mysore and Vijayapura. In each district ten plots were selected to know the incidence, symptoms and severity of the rhizome rot/ tip over disease of banana. In each plot random samples were made, from which the number of plants affected over the total number of plants was counted and expressed as per cent disease incidence using the formula.

\section{No. of infected plants \\ Per cent disease incidence $=-\times 100$ \\ Total No. of plants}

During the survey, observations were recorded with respect to previous crop, irrigation type, soil type, cultivar, planting material used such as tissue culture or sucker raised plants, date/month of planting, types of symptoms, agronomic practices and protection measures. Samples were collected for isolation of the pathogens and cultures of different isolates were preserved in the Department of Plant Pathology, College of Horticulture.

\section{Results and Discussion}

Roving survey was carried out in banana growing tracts of Karnataka in different districts viz., Bagalkot, Belagavi, Bidar, Dharwad, Gadag, Haveri, Koppal, Mysore and Vijayapura, to assess the incidence and severity of the disease during 2015 -2016. In each district ten plots infected with tip over disease were selected. During survey, symptoms were observed, disease incidence was recorded and infected samples were collected for further investigations.

Results of the survey revealed that, disease was noticed in all the locations with varied incidence levels. The disease incidence was found to be the highest in Bagalkot district (49.67\%) followed by Belagavi (34.16\%), Dharwad (25.75\%), Vijayapura (24.81\%), Bidar (15.75\%), Haveri (13.24\%), Koppal (11.07\%), Mysore (10.72\%) and the least was noticed in Gadag district (8.71\%). Among all the surveyed places in Karnataka maximum disease incidence was noticed in Kamalnagar village of Bidar district (99.27\%) followed by Sulla village of Bagalkot district $(95.00 \%)$ and Inchigeri village of Vijayapur district $(95.00 \%)$. The lowest incidence was observed in Hoovinahal village of Koppal taluk $(0.40 \%)$ followed by Hampiholi village in Ramdurg taluk $(0.50 \%)$. In some orchards of Mysore $(0.00 \%$ in Gundlupet and Shambudevanapura villages) and Vijayapura $(0.00 \%$ in Inchigeri and Kannur villages) districts no disease incidence of tip over/ rhizome rot of banana was recorded. These areas were found to be free from tip over or rhizome rot of banana.

Survey conducted in Bagalkot district revealed that all the fields planted with tissue culture plantlets had the incidence of tip over disease. The overall tip over incidence in Bagalkot district ranged from 12.50 to 95.00 per cent and has recorded the highest incidence $(49.67 \%)$ among all the surveyed districts in Karnataka. Among the different places in Bagalkot district, maximum tip over incidence was noticed in Sulla village $(95.00 \%)$ on three month old tissue cultured plantlets of Grand 
Naine (G-9) cultivar followed by Neeralkeri village $(90.00 \%)$ on two month old plantlets of Grand Naine cultivar. The least incidence was observed in Hiresellikeri village (12.50\%) on six months old Grand Naine plantlets followed by Udagatti village $(75.00 \%)$ on nine months old Grand Naine cultivar.

Survey conducted in Belagavi district has noticed the second highest tip over incidence among the surveyed districts recording 34.16 per cent. The overall tip over disease incidence ranged from 0.50 to 75.00 per cent. Among the different villages the highest disease incidence of tip over was observed in Kankanwadi recording 75.00 per cent on five month, 66.66 per cent on five month and 66.66 per cent on eight month old tissue culture raised Grand Naine cultivar respectively. The least incidence was observed in Hampiholi village $(0.50 \%)$ on twelve months old Grand Naine plantlets followed by Sureban village $(0.75 \%)$ on twenty months old Grand Naine cultivar.

In Bidar district the tip over disease incidence recorded was 15.75 per cent. The disease incidence ranged from 1.00 to 99.27 per cent. Among the villages surveyed in Bidar district, Kamalnagar village has shown the highest disease incidence (99.27\%) among all the surveyed villages in Karnataka on forty eight year old orchard of Grand Naine cultivar followed by Gumma village (12.70\%) on six month old tissue culture raised Grand Naine cultivar. The least per cent incidence (1.00\%) was noticed on six month old Grand Naine cultivar in Kamalnagar village followed by Gunnalli village $(1.55 \%)$ on five month old sucker raised Grand Naine cultivar.

The tip over disease incidence recorded in Dharwad district was 25.75 per cent which is the third highest incidence among the surveyed districts in Karnataka. The disease incidence ranged from12.50 to 45.00 per cent.
In Dharwad taluk, maximum per cent disease incidence was noticed in Uppinbetageri village $(45.00 \%)$ on three month old Grand Naine cultivar followed by Ambalikoppa village $(42.50 \%)$ on two month old Grand Naine cultivar. The least incidence was observed in Veerapur village $(12.50 \%)$ on six month old Grand Naine cultivar followed by in Agasanahalli village (15.00\%) on six month old Grand Naine cultivar.

The results of the roving survey of Gadag district revealed that the disease incidence recorded as 8.71 per cent. The per cent disease incidence of Gadag district ranged from 1.00 to 40.00 per cent. Among the villages surveyed, Inamvenkatapur has shown the highest per cent disease incidence $(40.00 \%)$ on six month old tissue culture raised Grand Naine cultivar followed by one more plot in Inamvenkatapur by recording 20.00 per cent incidence. The lowest tip over incidence was observed one plot in Inamvenkatapur (1.00\%) on three month old tissue culture raised Grand Naine cultivar and three plots in Hangankatti $(1.00 \%)$ on six, four and six month old Grand Naine cultivar.

The tip over disease incidence recorded in Haveri district was 13.24 per cent. The average disease incidence in Haveri district ranged from 1.33 to 34.96 per cent. Among the villages surveyed, Balembida has shown the highest tip over incidence $(34.96 \%)$ on eleven month old Grand Naine cultivar followed by Nilogal village $(25.00 \%)$ on twelve month old Grand Naine cultivar. The lowest incidence was observed in Halekoti village $(1.33 \%)$ on seven month old Grand Naine cultivar followed by Akkialur and Kadarmandalagi villages $(5.00 \%)$ on twelve month old Grand Naine cultivar.

Survey in Koppal district revealed that the tip over disease incidence was to the tune of recorded 11.07 per cent. 
Table.1 Incidence of tip over disease in major banana growing districts of Karnataka during 2015-16

\begin{tabular}{|c|c|c|c|c|c|c|c|c|c|c|c|c|c|c|c|}
\hline \multirow[t]{11}{*}{1} & 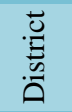 & $\begin{array}{l}\text { Total number } \\
\text { of plots }\end{array}$ & $\begin{array}{l}\text { Name of the } \\
\text { place }\end{array}$ & Variety & $\begin{array}{l}\text { Date of } \\
\text { planting }\end{array}$ & $\begin{array}{l}\text { Crop age } \\
\text { (months) }\end{array}$ & Soil type & & $\begin{array}{l}\text { ious } \\
\text { op }\end{array}$ & Irri & tion & & nter & $\begin{array}{c}\text { Protection measures/ Agronomic } \\
\text { practices }\end{array}$ & $\begin{array}{l}\text { Disease } \\
\text { incidence } \\
(\%)\end{array}$ \\
\hline & \multirow{10}{*}{ 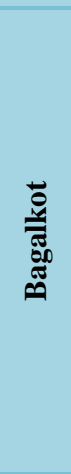 } & 1 & Badami & \multirow[t]{2}{*}{ Grand Naine } & July, 2015 & 3 & $\begin{array}{l}\text { Black } \\
\text { Sandy }\end{array}$ & \multicolumn{2}{|c|}{ Sugarcane } & \multicolumn{2}{|c|}{ Drip } & \multicolumn{2}{|r|}{-} & FYM, Bavistin & 40.00 \\
\hline & & 2 & Sulla & & June 2015 & 3 & Red Sandy & \multicolumn{2}{|c|}{ Sugarcane } & \multicolumn{2}{|c|}{ Drip } & \multicolumn{2}{|r|}{-} & FYM, Bavistin DM45, & 95.00 \\
\hline & & 3 & Hiresellikeri & \multirow[t]{3}{*}{ Grand Naine } & Feb, 2015 & 6 & Red soil & \multicolumn{2}{|c|}{ Maize } & \multicolumn{2}{|c|}{ Drip } & \multicolumn{2}{|r|}{-} & FYM, Chemycin & 12.50 \\
\hline & & 4 & Neeralkeri & & Sep, 2015 & 2 & Red Sandy & \multicolumn{2}{|c|}{ Sugarcane } & \multicolumn{2}{|c|}{ Drip } & \multicolumn{2}{|c|}{ Marigold } & Neemcake, Rogor, Chlorothalonil & 90.00 \\
\hline & & 5 & Shiroor & & Feb, 2015 & 6 & Red Sandy & \multicolumn{2}{|c|}{ Cowpea } & \multicolumn{2}{|c|}{ Flood } & \multicolumn{2}{|c|}{-} & FYM & 50.00 \\
\hline & & 6 & Sokanadagi & Rajapuri & Apr, 2015 & 2 & Black & Sug & cane & & & & - & FYM, DM45, Cowdung & 41.66 \\
\hline & & 7 & & & Mar, 2015 & 2 & Black & Brir & & & & & - & & 33.33 \\
\hline & & 8 & Udagatti & Grand Naine & Jun, 2015 & 3 & Black & Mai & & & & & - & FYM & 75.00 \\
\hline & & 9 & Sunaga & Rajapuri & July, 2016 & 1 & Red Sandy & Sug & cane & & & & - & $\begin{array}{l}\text { FYM, Streptocycline + COC, } \\
\text { Banana special }\end{array}$ & 19.23 \\
\hline & & 10 & & & Sep, 2016 & 1 & Red Sandy & Sug & cane & & & & nion & FYM, 19:19:19 & 40.00 \\
\hline & & & & & & Dis & ict mean & & & & & & & & 49.67 \\
\hline & & Contd..... & & & & & & & & & & & & & \\
\hline & 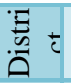 & $\begin{array}{l}\text { Total number of } \\
\text { plots }\end{array}$ & $\begin{array}{l}\text { Name of the } \\
\text { place }\end{array}$ & Variety & $\begin{array}{l}\text { Date of } \\
\text { planting }\end{array}$ & $\begin{array}{l}\text { Crop as } \\
\text { (month }\end{array}$ & Soil t & & $\begin{aligned} \text { Pre } \\
\mathrm{c}\end{aligned}$ & & Irrig & & $\begin{array}{l}\text { Inter } \\
\text { crop }\end{array}$ & $\begin{array}{c}\text { Protection measures/ Agronomic } \\
\text { practices }\end{array}$ & $\begin{array}{c}\text { Disease } \\
\text { incidence }(\%)\end{array}$ \\
\hline & & 1 & Chikkatadasi & Grand Naine & Jul, 2015 & 6 & Red c & & & & $\mathrm{D}$ & & - & FYM & 20.83 \\
\hline & & 2 & Hampiholi & & Dec, 2014 & 13 & later & & & & $\mathrm{D}$ & & - & Neem cake, FYM, Tilt & 30.00 \\
\hline & & 3 & & & Jan, 2015 & 12 & Red c & & & & Flc & & - & Boran, FYM, Potash & 0.50 \\
\hline & & 4 & Sureban & & Jan,2013 & 20 & Red sa & & & & $\mathrm{D}$ & & - & Asafoetida, Neemcake & 0.75 \\
\hline & & 5 & Kankanwadi & Grand Naine & Sep, 2015 & 5 & Black s & ndy & Sug & & $\mathrm{D}$ & & - & Cowurine, tilt & 66.66 \\
\hline & & 6 & & & Feb, 2015 & 9 & Black s & ndy & Sug & & $\mathrm{D}$ & & - & Neemcake & 35.00 \\
\hline & 㿿 & 7 & & & Sep, 2014 & 17 & Black s & ndy & suga & & $\mathrm{D}$ & & - & $\begin{array}{l}\text { Neemcake, Phorate, Tilt, Banana } \\
\text { special, Potash, Trichoderma, } \mathrm{Zn}\end{array}$ & 36.25 \\
\hline & & 8 & & & Dec, 2014 & 12 & Black s & ndy & Sug & & $\mathrm{D}$ & & Marigold & & 10.00 \\
\hline & & 9 & & & Aug, 2015 & 5 & Red sa & & $\begin{array}{l}\text { Bete } \\
\text { cap }\end{array}$ & & D & & - & $\begin{array}{l}\text { Vermicompost, FYM, Ridomil, } \\
\text { Karate, COC, Trichoderma, } \\
\text { Chloropyriphos }\end{array}$ & 75.00 \\
\hline & & 10 & & & June, 2015 & 8 & Black s & ndy & $\begin{array}{r}\text { Turn } \\
\text { Cak }\end{array}$ & & $\mathrm{D}$ & & Marigold & Neemcake, Phorate & 66.66 \\
\hline & & & & & & & strict Mean & & & & & & & & 34.16 \\
\hline
\end{tabular}


Contd.

\begin{tabular}{|c|c|c|c|c|c|c|c|c|c|c|c|c|}
\hline \multirow[t]{11}{*}{3} & $\stackrel{\vec{B}}{\mathscr{A}}$ & No. of plots & $\begin{array}{l}\text { Name of the } \\
\text { places }\end{array}$ & Variety & $\begin{array}{l}\text { Date of } \\
\text { planting }\end{array}$ & $\begin{array}{l}\text { Crop age } \\
\text { (months) }\end{array}$ & Soil type & $\begin{array}{l}\text { Previous } \\
\text { crop }\end{array}$ & Irrigation & $\begin{array}{l}\text { Inter } \\
\text { crop }\end{array}$ & $\begin{array}{l}\text { Protection measures/ } \\
\text { Agronomic practices }\end{array}$ & $\begin{array}{c}\text { Disease } \\
\text { incidence }(\%)\end{array}$ \\
\hline & \multirow{10}{*}{ 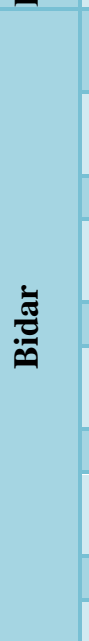 } & 1 & \multirow[t]{3}{*}{ Kamalnagar } & \multirow[t]{3}{*}{ Grand Naine } & Apr, 2014 & 48 & Lake soil & $\begin{array}{l}\text { Black } \\
\text { gram }\end{array}$ & Drip & - & FYM, Mancozeb & 99.27 \\
\hline & & 2 & & & $\begin{array}{l}\text { May, } \\
2015\end{array}$ & 10 & $\begin{array}{l}\text { Black wet } \\
\text { soil }\end{array}$ & $\begin{array}{l}\text { Black } \\
\text { gram }\end{array}$ & Drip & - & FYM & 5.00 \\
\hline & & 3 & & & Apr, 2016 & 6 & Black clay & Soybean & Flood & - & FYM, 19:19:19 & 1.00 \\
\hline & & 4 & \multirow[t]{3}{*}{ Chitta } & \multirow[t]{7}{*}{ Grand Naine } & Apr, 2015 & 6 & Red sandy & Sugarcane & Drip & Chilli & $\begin{array}{l}\text { FYM, Banana special, } \\
\text { Carbendazim }\end{array}$ & 11.66 \\
\hline & & 5 & & & Apr, 2015 & 11 & Red sandy & Sugarcane & Drip & Papaya & Organic & 2.00 \\
\hline & & 6 & & & Mar, 2015 & 11 & $\begin{array}{l}\text { Clay } \\
\text { sandy }\end{array}$ & $\begin{array}{l}\text { Black } \\
\text { gram }\end{array}$ & Flood & - & $\begin{array}{l}\text { FYM, Banana special, } \\
\text { Chlorothalonil }\end{array}$ & 6.89 \\
\hline & & 7 & Goranahalli & & Mar, 2015 & 7 & Black & Red Gram & Drip & - & Neemcake & 12.50 \\
\hline & & 8 & Gunnalli & & $\begin{array}{l}\text { May, } \\
2016\end{array}$ & 5 & $\begin{array}{l}\text { Red sandy } \\
\text { with clay }\end{array}$ & Red gram & Drip & Chilli & $\begin{array}{l}\text { Neem cake, FYM, } \\
\text { Banana special, 19:19:19 }\end{array}$ & 1.55 \\
\hline & & 9 & Gumma & & Mar, 2015 & 6 & Red sandy & Red gram & Drip & - & FYM, Neemcake & 12.70 \\
\hline & & 10 & & & Mar, 2015 & 6 & Red sandy & Sugarcane & Drip & \multirow[t]{2}{*}{-} & & 5.00 \\
\hline \multicolumn{11}{|c|}{ District Mean } & & 15.75 \\
\hline \multicolumn{13}{|c|}{ Contd..... } \\
\hline \multirow[t]{11}{*}{4} & 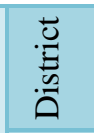 & $\begin{array}{c}\text { Total } \\
\text { Number } \\
\text { of plots }\end{array}$ & $\begin{array}{l}\text { Name of the } \\
\text { places }\end{array}$ & Variety & $\begin{array}{l}\text { Date of } \\
\text { planting }\end{array}$ & $\begin{array}{l}\text { Crop age } \\
\text { (months) }\end{array}$ & Soil type & $\begin{array}{l}\text { Previous } \\
\text { crop }\end{array}$ & Irrigation & $\begin{array}{l}\text { Inter } \\
\text { crop }\end{array}$ & $\begin{array}{l}\text { Protection measures/ } \\
\text { Agronomic practices }\end{array}$ & $\begin{array}{c}\text { Disease } \\
\text { incidence } \\
(\%)\end{array}$ \\
\hline & \multirow{10}{*}{ 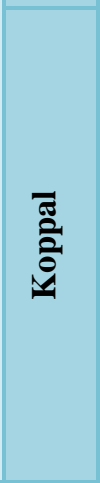 } & 1 & Abbigeri & \multirow[t]{2}{*}{ Grand Naine } & Jan, 2015 & 2 & Black & Maize & Drip & - & Neemcake, COC & 3.33 \\
\hline & & 2 & Hoovinahal & & Feb, 2015 & 1 & Red sandy & Sugarcane & Drip & - & Phorate, FYM, Neemcake & 0.40 \\
\hline & & 3 & Hireshindagi & Rajapuri & Dec, 2014 & 3 & Red sandy & Beans & Flood & - & Neemcake, FYM, Phorate & 5.00 \\
\hline & & 4 & Mattur & Grand Naine & Jun, 2014 & 9 & Black & Sugarcane & Drip & - & Neemcake & 8.00 \\
\hline & & 5 & Kinnal & & Sept, 2014 & 5 & Red sandy & Sugarcane & Drip & - & $\begin{array}{l}\text { FYM, } \\
\text { Humic acid }\end{array}$ & 7.50 \\
\hline & & 6 & Gajendragad & Grand Naine & Nov, 2014 & 4 & Red sandy & Sugarcane & Flood & - & FYM & 50.00 \\
\hline & & 7 & Hanumsagar & & Dec, 2014 & 3 & Black & Maize & Drip & - & FYM & 20.00 \\
\hline & & 8 & Hiremannapur & & Oct, 2014 & 5 & Red sandy & Sugarcane & Flood & - & FYM & 10.00 \\
\hline & & 9 & Tavaregere & & Sept, 2014 & 6 & Red sandy & Maize & Drip & - & FYM, Phorate & 1.50 \\
\hline & & 10 & Yelburti & Rajapuri & July, 2014 & 8 & Red sandy & Sugarcane & Drip & - & FYM, Phorate & 5.00 \\
\hline & & & & & & District & Mean & & & & & 11.07 \\
\hline
\end{tabular}


Int.J.Curr.Microbiol.App.Sci (2018) 7(7): 1337-1347

Contd.....

\begin{tabular}{|c|c|c|c|c|c|c|c|c|c|c|c|c|}
\hline \multirow[t]{11}{*}{5} & 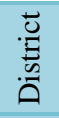 & $\begin{array}{l}\text { Total Number } \\
\text { of plots }\end{array}$ & $\begin{array}{l}\text { Name of } \\
\text { the place }\end{array}$ & Variety & $\begin{array}{l}\text { Date of } \\
\text { planting }\end{array}$ & $\begin{array}{l}\text { Crop age } \\
\text { (months) }\end{array}$ & Soil type & $\begin{array}{l}\text { Previous } \\
\text { crop }\end{array}$ & Irrigation & Intercrop & $\begin{array}{c}\text { Protection measures/ Agronomic } \\
\text { practices }\end{array}$ & $\begin{array}{l}\text { Disease } \\
\text { inciden } \\
\text { ce }(\%)\end{array}$ \\
\hline & \multirow{10}{*}{ 莺 } & 1 & Horti & \multirow[t]{3}{*}{ Grand Naine } & Jul, 2015 & 6 & Red & Wheat & Drip & - & Vermicompost Neemcake & 2.50 \\
\hline & & 2 & \multirow[t]{3}{*}{ Inchigeri } & & Aug, 2015 & 3 & Clay & Wheat & Drip & $\begin{array}{c}\text { Ground } \\
\text { nut }\end{array}$ & FYM & 75.00 \\
\hline & & 3 & & & Apr, 2015 & 6 & Clay, & Red gram & Drip & - & - & 95.00 \\
\hline & & 4 & & Rajapuri & Dec, 2015 & 6 & Black & - & Drip & - & Chicken manure, Blitox, DM45 & 0.00 \\
\hline & & 5 & \multirow[t]{3}{*}{$\begin{array}{l}\text { Babaleshw } \\
\text { ar }\end{array}$} & \multirow[t]{2}{*}{ Grand Naine } & Oct, 2014 & 12 & Red sandy & Grape & Drip & - & $\begin{array}{l}\text { Phorate, FYM, Humic acid, } \\
\text { Cowdung }\end{array}$ & 31.25 \\
\hline & & 6 & & & Nov, 2014 & 12 & Red sandy & Grape & Drip & - & $\begin{array}{l}\text { FYM, Neemcake, Humic acid, } \\
\text { Bavistin }\end{array}$ & 21.05 \\
\hline & & 7 & & Rajapuri & Apr, 2015 & 7 & Black clay & Ground nut & Basin & Onion & FYM & 15.00 \\
\hline & & 8 & \multirow[t]{3}{*}{ Kannur } & \multirow[t]{2}{*}{ Grand Naine } & Apr, 2015 & 12 & Black & Maize & Drip & Onion & $\begin{array}{l}\text { Neemcake, FYM, Cowdung } \\
\text { slurry, Phorate, Trichoderma, } \\
\mathrm{MgSo}_{4}\end{array}$ & 3.33 \\
\hline & & 9 & & & Jun, 2015 & 7 & Red sandy & Tomato & Drip & Onion & $\begin{array}{l}\text { Neemcake, FYM, Bavistin, } \\
\text { Cowdung }\end{array}$ & 0.00 \\
\hline & & 10 & & Grand Naine & Apr, 2015 & 8 & Black clay & Red gram & Drip & - & FYM, Phorate, Blitox & 5.00 \\
\hline
\end{tabular}

\begin{tabular}{|c|c|c|c|c|c|c|c|c|c|c|c|c|}
\hline & & Contd.... & & & & & & & & & & \\
\hline \multirow[t]{11}{*}{6} & : & $\begin{array}{c}\text { Total } \\
\text { Number of } \\
\text { plots }\end{array}$ & Name of the place & Variety & $\begin{array}{l}\text { Date of } \\
\text { planting }\end{array}$ & $\begin{array}{l}\text { Crop age } \\
\text { (months) }\end{array}$ & Soil type & $\begin{array}{l}\text { Previous } \\
\text { crop }\end{array}$ & Irrigation & Intercrop & $\begin{array}{l}\text { Protection measures/ } \\
\text { Agronomic practices }\end{array}$ & $\begin{array}{c}\text { Disease incidence } \\
(\%)\end{array}$ \\
\hline & \multirow{10}{*}{ 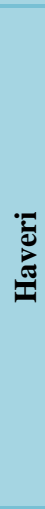 } & 1 & Agasanahalli & \multirow[t]{4}{*}{ Grand Naine } & Mar, 2015 & 5 & Black & - & Drip & Arecanut & $\begin{array}{l}\text { Dried leaf mulch, FYM, } \\
\text { Phorate, Blitox, }\end{array}$ & 6.66 \\
\hline & & 2 & \multirow[t]{3}{*}{ Kadaramandalagi } & & Mar, 2015 & 12 & Black & Maize & Drip & - & FYM, Phorate, & 5.00 \\
\hline & & 3 & & & June, 2016 & 2 & Black & Sugarcane & Drip & - & \multirow[t]{2}{*}{ Chilli thumb mulch, FYM } & 15.00 \\
\hline & & 4 & & & June, 2016 & 2 & Black & Sugarcane & Drip & - & & 15.00 \\
\hline & & 5 & \multirow[t]{2}{*}{ Akkialur } & \multirow[t]{5}{*}{ Grand Naine } & June, 2016 & 2 & Red & Maize & Drip & Arecanut & FYM, Phorate & 18.51 \\
\hline & & 6 & & & June, 2015 & 12 & Black & Sugarcane & Drip & - & Goat manure, Phorate & 5.00 \\
\hline & & 7 & Balembida & & Sept, 2015 & 11 & Black & Sugarcane & Drip & Arecanut & FYM & 34.96 \\
\hline & & 8 & Halekoti & & Feb, 2016 & 7 & Black & Maize & Drip & - & FYM, Banana special & 1.33 \\
\hline & & 9 & Hiroor & & Mar, 2016 & 5 & Clay & Paddy & Drip & Arecanut & Goat manure & $5.90^{\circ}$ \\
\hline & & 10 & Nilogal & Grand Naine & May, 2015 & 12 & $\begin{array}{l}\text { Clay, } \\
\text { Black } \\
\text { Sandy }\end{array}$ & Cotton & Drip & - & FYM, Bavistin, Blitox, & 25.00 \\
\hline \multicolumn{12}{|c|}{ District Mean } & 13.24 \\
\hline
\end{tabular}

Contd.....

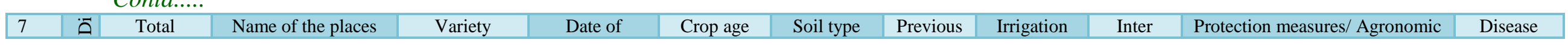


Int.J.Curr.Microbiol.App.Sci (2018) 7(7): 1337-1347

\begin{tabular}{|c|c|c|c|c|c|c|c|c|c|c|c|c|}
\hline & & $\begin{array}{l}\text { Number of } \\
\text { plots }\end{array}$ & & & planting & (months) & & crop & & crop & practices & $\begin{array}{l}\text { incidence } \\
(\%)\end{array}$ \\
\hline & & 1 & Gundlupet & Yelakki bale & Nov, 2015 & 3 & Red sandy & Tomato & Drip & $\begin{array}{l}\text { Water } \\
\text { melon, } \\
\text { Chilli }\end{array}$ & $\begin{array}{l}\text { Neem cake, Banana special, } \\
\text { chlorothalonil, COC+ bactinash, } \\
\text { Tilt, 19:19:19 }\end{array}$ & 0.00 \\
\hline & & 2 & Gungral Chatra & & Jun, 2015 & 8 & Red sandy & Tomato & Drip & - & Phorate, Neem cake, 19:19:19 & 15.00 \\
\hline & & 3 & & Grand Naine & Oct, 2015 & 3 & Red sandy & - & Drip & - & $\begin{array}{l}\text { Furadon, Humic acid, Neem } \\
\text { cake }\end{array}$ & 5.50 \\
\hline & & 4 & & Pacchabale & Jun, 2015 & 8 & Red sandy & Chilli & Drip & Chilli & Phorate & 5.00 \\
\hline & 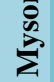 & & & Grand Naine & Mar, 2015 & 11 & Red sandy & $\begin{array}{l}\text { Tomato, } \\
\text { Chilli }\end{array}$ & Drip & \multirow[t]{2}{*}{ Chilli } & \multirow[t]{2}{*}{$\begin{array}{l}\text { Phorate, Neem cake, Rogor, } \\
\text { humic acid }\end{array}$} & 50.00 \\
\hline & & 5 & Hosakote & & & & & & & & & \\
\hline & & 6 & Kadakola & Yelakki bale & Sept, 2015 & 5 & Red sandy & Tomato & Drip & \multirow{2}{*}{$\begin{array}{l}- \\
-\end{array}$} & \multirow[t]{2}{*}{ Phorate, micronutrients } & 15.00 \\
\hline & & 7 & & Grand Naine & Oct, 2015 & 4 & Red sandy & Chilli & Drip & & & 5.00 \\
\hline & & 8 & Keelanapur & Yelakki bale & Mar, 2015, & 9 & Red sandy & - & Drip & - & Neem cake, Phorate & 7.50 \\
\hline & & 9 & Shambudevanapura & Yelakki bale & Sept, 2015 & 5 & Red sandy & Tomato & Drip & \multirow{2}{*}{$\begin{array}{c}- \\
\text { Chilli }\end{array}$} & \multirow[t]{2}{*}{ Neemcake, Phorate, 19:19:19 } & 4.16 \\
\hline & & 10 & & Pachhabale & Oct, 2015 & 4 & Red sandy & Tomato & Drip & & & 0.00 \\
\hline \multicolumn{12}{|c|}{ District mean } & 10.72 \\
\hline \multicolumn{13}{|c|}{ Contd..... } \\
\hline \multirow[t]{13}{*}{8} & & & Name of the place & Variety & Date of & Crop age & Soil type & Previous & Irrigation & Inter & Protection measures/ & Disease \\
\hline & 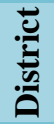 & $\begin{array}{c}\text { Total } \\
\text { Number } \\
\text { of plots }\end{array}$ & Village & & planting & (months) & & crop & & crop & Agronomic practices & $\begin{array}{l}\text { incidence } \\
(\%)\end{array}$ \\
\hline & \multirow{10}{*}{ 吾 } & 1 & Agasanahalli & \multirow[t]{2}{*}{ Grand Naine } & July, 2016 & 6 & Black & Sugarcane & Drip & \multirow{2}{*}{$\begin{array}{l}- \\
-\end{array}$} & FYM & 15.00 \\
\hline & & 2 & Ambalikoppa & & Oct, 2016 & 2 & Black & Maize & Drip & & - & 42.50 \\
\hline & & 3 & Chikkamalligwad & Rajapuri & July, 2016 & 6 & Black & Sugarcane & Flood & - & Bavistin, FYM & 30.00 \\
\hline & & 4 & Dandikoppa & Grand Naine & Aug, 2016 & 4 & Black & Beans & Drip & \multirow{2}{*}{$\begin{array}{l}- \\
-\end{array}$} & FYM & 25.00 \\
\hline & & 5 & Dasanakoppa & & Mar, 2016 & 10 & Black & Maize & Drip & & FYM, Chloropyriphos & 16.00 \\
\hline & & 6 & Mavinakoppa & & July, 2016 & 6 & Black & Maize & Drip & - & - & 25.00 \\
\hline & & 7 & Uppinbetageri & & Sep, 2016 & 3 & Black & Sugarcane & Flood & - & FYM & 45.00 \\
\hline & & 8 & Veerapur & & July, 2016 & 6 & Black & Sugarcane & Drip & - & FYM, Neemcake & 12.50 \\
\hline & & 9 & Gudisagar & Grand Naine & $\begin{array}{l}\text { May, } \\
2016\end{array}$ & 8 & Red Sandy & Sugarcane & Drip & - & - & 21.53 \\
\hline & & 10 & & & Sep, 2016 & 3 & Red loam & Sugarcane & Drip & - & Vermicompost, Bavistin & 25.00 \\
\hline & & & & & & District $\mathrm{N}$ & & & & & & 25.75 \\
\hline
\end{tabular}

Contd...... 
Int.J.Curr.Microbiol.App.Sci (2018) 7(7): 1337-1347

\begin{tabular}{|c|c|c|c|c|c|c|c|c|c|c|c|c|}
\hline \multirow[t]{11}{*}{9} & 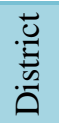 & $\begin{array}{c}\text { Total } \\
\text { Number } \\
\text { of plots }\end{array}$ & $\begin{array}{c}\text { Name of the } \\
\text { place }\end{array}$ & Variety & $\begin{array}{l}\text { Date of } \\
\text { planting }\end{array}$ & $\begin{array}{l}\text { Crop age } \\
\text { (months) }\end{array}$ & Soil type & $\begin{array}{l}\text { Previous } \\
\text { crop }\end{array}$ & Irrigation & $\begin{array}{l}\text { Protection measures/ } \\
\text { Agronomic practices }\end{array}$ & $\begin{array}{l}\text { Inter } \\
\text { crop }\end{array}$ & $\begin{array}{c}\text { Disease } \\
\text { incidence } \\
(\%)\end{array}$ \\
\hline & \multirow{10}{*}{ 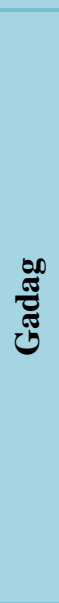 } & 1 & \multirow[t]{5}{*}{ Inamvenkatapur } & \multirow[t]{5}{*}{ Grand Naine } & $\begin{array}{l}\text { Sept, } \\
2014\end{array}$ & 6 & Red Sandy & $\begin{array}{l}\text { Green } \\
\text { gram }\end{array}$ & Drip & $\begin{array}{l}\text { Chloropyriphos, } \\
\text { Bavistin, Neem oil, } \\
\text { 19:19:19 }\end{array}$ & - & 8.81 \\
\hline & & 2 & & & $\begin{array}{l}\text { Sept, } \\
2014\end{array}$ & 6 & Red Sandy & $\begin{array}{l}\text { Ground } \\
\text { nut }\end{array}$ & Drip & FYM & - & 40.00 \\
\hline & & 3 & & & Oct, 2014 & 5 & Red Sandy & Maize & Flood & - & - & 20.00 \\
\hline & & 4 & & & Aug, 2014 & 6 & Red Sandy & Coriander & Drip & $\begin{array}{l}\text { FYM, Tilt, } \\
\text { Chloropyriphos }\end{array}$ & - & 6.81 \\
\hline & & 5 & & & Oct, 2015 & 3 & Red Sandy & Maize & Drip & - & - & 1.00 \\
\hline & & 6 & \multirow[t]{5}{*}{ Hangankatti } & \multirow[t]{5}{*}{ Grand Naine } & Sep, 2014 & 6 & Red Sandy & Soybean & Drip & Saaf & - & 1.00 \\
\hline & & 7 & & & Oct, 2014 & 4 & Red Sandy & Maize & Drip & Chloropyriphos & Tomato & 1.00 \\
\hline & & 8 & & & Jan, 2015 & 1 & Red Sandy & Maize & Drip & Chloropyriphos, Blitox & - & 2.50 \\
\hline & & 9 & & & Sep, 2014 & 6 & Red Sandy & - & Flood & Chloropyriphos, Blitox & - & 1.00 \\
\hline & & 10 & & & Oct, 2014 & 4 & Red Sandy & - & Drip & FYM, Blitox & - & 5.00 \\
\hline
\end{tabular}


The disease incidence in Koppal district ranged from 0.40 to 50.00 per cent. Among the surveyed villages in Koppal district, Gajendragad village has recorded the highest per cent disease incidence $(50.00 \%)$ on four month old Grand Naine cultivar followed by Hanumsagar village $(20.00 \%)$ on three month old Grand Naine cultivar. The least disease incidence was observed in Hoovinahal village $(0.40 \%)$ on one month old Grand Naine cultivar as it was newly planted orchard even though symptoms like browning of tip were observed which was followed by Tavaregere village $(1.50 \%)$ on six month old Grand Naine cultivar.

The disease incidence recorded in Mysore district was 10.72 per cent. The disease incidence ranged from 4.16 to 50.00 . Among the villages surveyed in Mysore district, Hosakote village has shown maximum disease incidence $(50.00 \%)$ on eleven month old Grand Naine cultivar followed by Gungral chatra $(15.00 \%)$ on sucker raised eight month old Yelakki bale cultivar and Kadakola $(15.00 \%)$ on five month old sucker raised Yelakki bale cultivar. The lowest incidence was observed in villages of Gundlupet $(0.00 \%)$ on three month old Yelakki bale cultivar and Shambudevanapura $(0.00 \%)$ on four month old Pachhabale cultivar followed by another plot in Shambudevanapura $(4.16 \%)$ on five month old Yelakki bale cultivar.

The tip over incidence of the Vijayapura district has shown the fourth highest incidence among all the surveyed districts recording 24.81 per cent. The tip over incidence in Vijayapura district ranged from 0.00 to 95.00 per cent. Maximum per cent disease incidence was observed in Inchigeri village (95.00\%) on six month old plantlets of Grand Naine cultivar followed by another plot in Inchigeri village $(75.00 \%)$ on three month old plantlets Grand Naine cultivar. No disease incidence was noticed in Kannur village $(0.00 \%)$ on seven month old Grand Naine cultivar and in Inchigeri village $(0.00 \%)$ on six month old Rajapuri cultivar followed by Horti village (2.50\%) on six month old Grand Naine cultivar.

The results of the roving survey revealed that the disease incidence was found to be maximum in tissue culture raised plantlets of Grand Naine cultivar in first two to six month crop stages. Observations recorded during the survey are depicted in table 1.

Survey was conducted to collect the information, record on the occurrence, incidence of plant diseases and assessment of losses from disease.

An intensive roving survey was carried out in nine districts of Karnataka, viz., Bagalkot, Belagavi, Bidar, Dharwad, Gadag, Haveri, Koppal, Mysore and Vijayapura during 201516 to know the disease incidence of tip rot or rhizome rot of banana. The districts were selected based on maximum area of banana production and occurrence of tip over disease incidence. The data on survey revealed that, the disease incidence varied from locality to locality, tissue cultured plantlets or sucker raised and environmental conditions. Most of the farmers were using tissue culture plantlets, following drip irrigation system, no pre treatment to the suckers, almost maximum incidence was noticed in summer months which have created congenial micro-climate for disease development and the pathogen can survive in high temperature range with high multiplication rate.

Total plots surveyed were 90, among them no disease incidence was observed in four plots viz., two plots in Mysore districs (Gundlupet and Shambudevanapura) and two plots in Vijayapura (Inchigeri and Kannur). This may be because use of good planting materials 
(mostly with sucker raised) and the management practices with respect to maintenance of orchard and pre treatments before planting in case of tissue cultured plantlets. The incidence of tip over was noticed in all the remaining 86 plots which ranged from 0.40 in Hoovinahal village of Koppal taluk to 99.27 per cent in Kamalnagar village in Bidar district. Among the districts surveyed average tip over disease incidence was noticed to be the highest in Bagalkot district (49.67\%) followed by Belagavi (34.16\%), Dharwad (25.75\%), Vijayapura $(24.18 \%)$ districts. The least disease incidence was observed in Gadag $(8.71 \%)$ followed by Mysore $(10.72 \%)$ districts. Almost all the surveyed districts were using tissue culturally propagated planting materials. Hence, these plots were more susceptible to tip over disease than sucker raised plants. But in Mysore district maximum plots were sucker raised and the incidence was less as compared to northern Karnataka and also with the prevailing environmental conditions being less congenial for disease development. In all areas the incidence was maximum at initial months from one month to six months crop stage.

In India the first report of this disease was from Tamilnadu as bacterial corm rot of nendran banana by $E$. carotovora which has destroyed maximum area under cultivation (Bhakthavathsalu et al., 1976). Chattopadhyay and Mukherjee (1986) detected a pseudostem rot of banana due to $E$. chrysanthemi pv. parasidiaca for the first time on new plantation of Cavendish banana at B.S.K.V.V. in 1983. The present survey record is in line with the studies conducted by Snehalatharani (2001), who reported that the disease incidence was prevalent in southern parts of Karnataka viz., Bangalore, Tumkur and Kolar districts and West Godavari and Konnur districts of Andhra Pradesh. Nagaraj et al., (2002) conducted survey and reported the incidence of tip rot for the first time in Karnataka. Similar findings with the present survey in Belagavi district were reported by Thammaiah et al., (2005a) who noticed that the incidence of bacterial rhizome rot was 12.0 per cent in Raibagh and Gokak.

Findings were in conformity with Vijayalaxmi (2012), who has conducted an intensive roving survey during 2011-2012 to record the tip rot incidence in Northern parts of Karnataka and reported that the disease incidence was noticed in all the locations surveyed, in a range from 4.25 to 65.28 per cent. Maximum disease incidence was 40.37 per cent recorded in Bijapur district followed by Bagalkot (23.72\%). Nagrale et al., (2013) reported the collar and rhizome rot disease for first time in farmer's field during 2006-07 and attracted the attention of banana growers.

On the recent study with the similar observations were recorded by Thiyagarajan, et al., 2017, which states that, among the different districts, highest tip rot incidence of 12.99 was recorded from Koppal followed by Shimoga $(9.15 \%)$ and Chitradurga $(6.75 \%)$. Lowest incidence of 3.22 per cent was recorded in Bellari district. Maximum tip rot incidence of 12.99 per cent was recorded from Muddaballi village of Koppal taluk.

Vasundhara and Thammaiah (2017), conducted a roving survey in major banana growing areas of Belgaum, Bagalkot and Bijapur districts of Northern Karnataka during 2012 and it revealed that the incidence of the disease ranged from zero to thirty per cent. The highest incidence of the disease was recorded in Salahalli $(30.00 \%)$ on Grand Naine followed by Dastikoppa $(24.39 \%)$ on Grand Naine cultivar. Hence, the present results shows are in conformity with the reports of these researchers by showing maximum incidence on Grand Naine and Rajapuri cultivars. 


\section{References}

Bhakthavathsalu, C. M., Sathiamorthy, S. and Chockalingam, S. 1976. Bacterial head rot - a new disease in Nendran banana. Unda, Vellore, India, Tamil Nadu, Department of Agriculture. CAB. Horticultural Abstracts, 14: 046-0165.

Chattopadhyay, P. K. and Mukherjee, N. 1986. A pseudostem rot of banana due to Erwinia chrysanthemi pv. paradisica. Curr. Sci., India. 55 (6): 789-790.

Nagaraj, M. S., Khan, A. N. A., Ravikumar, M. R., and Amarnanjundeswara, H. 2002. Management of tip over disease of banana. Global Conference on Banana and Plantain, Bangalore, India, p. 28-31.

Nagrale, D, T., Suresh, N., G. B., Shailesh, P., Gawande, Asit, K. M., and Satish A. R. 2013. Characterization of a bacterial collar and rhizome rot of banana (Musa paradisiaca) caused by strains of Erwinia chrysanthemi pv. paradisiaca. J. Appl. Nat. Sci., 5 (2): 435-441.

Snehalatharani, A. and A. N. A. Khan. 2009. Bio-control of tipover disease of banana. Ann. Pl. Prot. Sci., 17(1): 149- 151.

Snehalatharani, A. 2001. Studies on etiology and biocontrol of tip-over disease of banana. M. Sc. (Agri.) Thesis, Uni. Agric. Sci., Bangalore, Karnataka (India).

Thammaiah, N., Kulkarni, M. S., Srikant Kulkarni. and Kanamadi, V. C. 2005a.
Survey and surveillance of viral and bacterial disease of banana in Northern parts of Karnataka, National Symposium on crop disease management in dryland Agriculture held at MAU, Parbhani from 12-14 January 2005, pp. 84.

Thiyagarajan, V., Yenjerappa, S. T., Sunkad, G., Aswathanarayana, D. S., Karuppaiah, V. And Shaila, H. M. 2017. Survey on tip- over disease of banana caused by Erwinia carotovora subsp. carotovora (Jones) Holland in parts north eastern Karnataka, India. Int. J. Curr. Microbiol. App. Sci., 6 (6): 2973- 2976.

Vasundhara, B. A. and Thammaiah, N. 2017. In vitro evaluation of plant extracts against the growth of Erwinia chrysanthemi. The Bioscan, 12 (3): 1449-1455.

Vijayalaxmi, S. T. 2012. Studies on tip over disease of banana caused by Erwinia carotovora subsp. carotovora (Jones) Holland and its management. M. Sc. Thesis submitted to University of Agricultural Sciences, Dharwad, pp. 63.

Vijayalaxmi, S. T., Ravikumar, M. R., Benagi, V. I., Virupakshaprabhu, H., Wali, S.Y. K. N., Pawer and Lingaraju, S. 2014. Survey on the tip over disease of banana caused by Erwinia carotovora subsp. carotovora (Jones) Holland in Northern Karnataka. J. Agric. Sci., 27 (3): 353354.

\section{How to cite this article:}

N. Pradnyarani, M.S. Kulkarni, K.C. Kirankumar, R.K. Mesta, B. Jagadeesh and H. Rohan. 2018. Roving Survey for the Incidence of Tip Over Disease of Banana in Major Banana Growing Districts of Karnataka, India. Int.J.Curr.Microbiol.App.Sci. 7(07): 1337-1347. doi: https://doi.org/10.20546/ijcmas.2018.707.159 